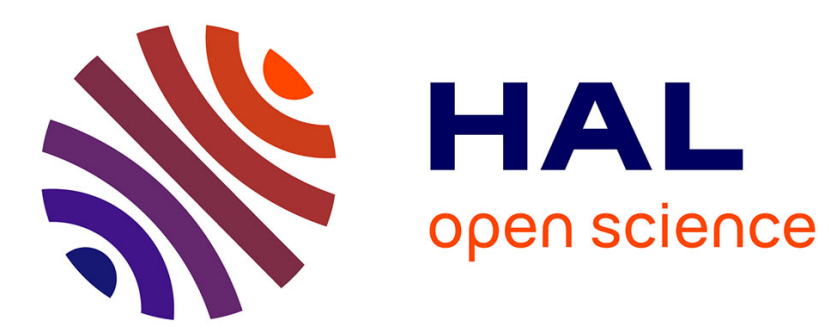

\title{
FPGA Architecture Support for Heterogeneous, Relocatable Partial Bitstreams
}

Christophe Huriaux, Olivier Sentieys, Russell Tessier

\section{To cite this version:}

Christophe Huriaux, Olivier Sentieys, Russell Tessier. FPGA Architecture Support for Heterogeneous, Relocatable Partial Bitstreams. FPL - 24th International Conference on Field Programmable Logic and Applications, Sep 2014, Munich, Germany. 10.1109/FPL.2014.6927494 . hal-01017184

\author{
HAL Id: hal-01017184 \\ https://hal.inria.fr/hal-01017184
}

Submitted on 2 Jul 2014

HAL is a multi-disciplinary open access archive for the deposit and dissemination of scientific research documents, whether they are published or not. The documents may come from teaching and research institutions in France or abroad, or from public or private research centers.
L'archive ouverte pluridisciplinaire HAL, est destinée au dépôt et à la diffusion de documents scientifiques de niveau recherche, publiés ou non, émanant des établissements d'enseignement et de recherche français ou étrangers, des laboratoires publics ou privés. 


\section{FPGA Architecture Support for Heterogeneous, Relocatable Partial Bitstreams}

\author{
Christophe Huriaux \\ University of Rennes 1 \\ IRISA \\ Lannion, France \\ christophe.huriaux@irisa.fr
}

\author{
Olivier Sentieys \\ Inria \\ IRISA \\ Lannion, France \\ olivier.sentieys@inria.fr
}

\author{
Russell Tessier \\ Department of Electrical and Computer Engineering \\ University of Massachusetts \\ Amherst, MA 01003 \\ tessier@umass.edu
}

\begin{abstract}
The use of partial dynamic reconfiguration in FPGA-based systems has grown in recent years as the spectrum of applications which use this feature has increased. For these systems, it is desirable to create a series of partial bitstreams which represent tasks which can be located in multiple regions in the FPGA fabric. While the transferal of homogeneous collections of lookup-table based logic blocks from region to region has been shown to be relatively straightforward, it is more difficult to transfer partial bitstreams which contain fixed-function resources, such as block RAMs and DSP blocks. In this paper we consider FPGA architecture enhancements which allow for the migration of partial bitstreams including fixed-function resources from region to region even if these resources are not located in the same position in each region. Our approach does not require significant, time-consuming place-and-route during the migration process. We quantify the cost of inserting additional routing resources into the FPGA architecture to allow for easy migration of heterogeneous, fixed-function resources. Our experiments show that this flexibility can be added for a relatively low overhead and performance penalty.
\end{abstract}

Keywords-Partial reconfiguration; FPGA; heterogeneous fixed-function blocks

\section{INTRODUCTION}

Almost since the creation of the first SRAM-based FPGAs there has been a desire to explore the benefits of partially reconfiguring a portion of an FPGA at run-time while the remainder of design functionality continues to operate uninterrupted. Recent advances in FPGA technology have fueled increased interest in the area from an application standpoint. Dynamic FPGA reconfiguration has been used successfully in applications such as communication coding [1], image processing [2], and networking [3], among others. In most cases, dynamic reconfiguration plays an important role in enhancing application performance, although reduced power consumption and increased fault tolerance are additional motivations.

Contemporary FPGA devices provide hardware interfaces which allow for portions of an FPGA to be modified at run-time without affecting the operation of unmodified areas. Although numerous papers have been written which examine region packing [3], software scheduling [4], and bitstream writing techniques [5] to facilitate partial reconfiguration, the approach is still used by designers on a limited basis.

Currently, the use of partial reconfiguration imposes limitations on the FPGA design: partially-reconfigurable regions must be constrained to a certain size and, in many cases, region bitstreams must be precompiled before application execution. In cases where a partial bitstream is translated from one region to another, the resources in both source and destination regions must match in terms of both functionality and the location of the resources (e.g. logic, fixed-function blocks) in the region.

In this paper, we develop an FPGA architecture which allows for easy translation of partial bitstreams (also called tasks) across partially-reconfigurable regions even if the relative placement of fixed-function blocks within the region is changed. This flexibility comes at the cost of isolating the routing resources from the fixed-function blocks. Our approach can be applied without the need for storage of numerous bitstreams for the same task or for the use of place-and-route tools when a task is moved from location to location.

Although our idea could be used for any island-style FPGA, the work presented in this paper has been specifically conducted in the context of the the European FP7 project FlexTiles [6], in which a dynamically reconfigurable logic fabric in a many-core architecture is used to accelerate specific functions. For this project, an architecture has been developed in which inputs and outputs in the fabric are made through dedicated fixed-function blocks inside the fabric, rather than I/Os spread in a ring around the fabric. This increases the importance of the free movement of tasks across partially-reconfigurable regions. As a result, it is necessary to translate tasks flexibly to locations on the fabric where the relative placement of fixed-function blocks (e.g. memory blocks, multipliers, I/Os) in the region is different.

The remainder of the paper is structured as follows. Section II presents previous work exploring the concept of partial reconfiguration including the relocation of tasks. In Section III, we present FPGA architectural enhancements which allow for the relocation of tasks with fixed-function blocks. In Section IV, our experimental methodology using a modified version of VPR 6.0 [7] is described. Experimental results are presented in Section V to quantify the area and performance overhead of the approach. Section VI concludes the paper and offers directions for future work.

\section{RELATED WORK}

The use of partial dynamic reconfiguration in FPGAs has been studied for over twenty years. Although early analysis primarily targeted Xilinx XC6200 series FPGAs [8], almost all recent work in the area has focused on Xilinx Virtex families 
and Altera Stratix V devices, which allow for partial reconfiguration of device regions. Most recent software systems in the area require the precompilation of task bitstreams [9][10] that are assigned to partially-reconfigurable regions (PRR). Occupancy in these locations can be managed by scheduling and region packing software [4].

In many cases it is desirable to place and route a task targeted to a PRR once and then to migrate the task to multiple locations on the FPGA die as needed. Often some routing is still needed to connect the newly placed region with surrounding interconnect. In Mignolet, et al [5], a fast router is used to connect a task to surrounding blocks. In Flynn, et al. [11], the routing of clock signals to the region is performed. If additional routing is performed during the merge, the migration of a task to a new PRR can be as long as 97 seconds [12], a prohibitive time for most applications.

PRRs are connected to static regions in recent Xilinx and Altera devices using lookup tables as an interface [9][10]. Older partial reconfiguration approaches overcome the interPRR communication issue by using a standard bus interface for communications between the PRR and the remainder of the circuit. Both Carver, et al. [13] and Tan and DeMara [14] use bus macros at fixed locations to allow a task to be located at a variety of positions within an FPGA. In some cases for the Virtex architecture, it is necessary to make bitstream modifications to the stored task (such as a new CRC) if it is moved to a different on-chip location, even if the logic resources in the original and destination locations exactly match. Touiza, et al. [15] provide a high-throughput method for moving a task from one region to another with a single bitstream by calculating most of the needed changes offline.

Two previous partial FPGA reconfiguration schemes directly address the issue of migrating a task which contains fixed-function blocks. Krasteva, et al. [16] examine techniques which move a task with fixed-function blocks to a new region where the blocks are in the same relative position in the region. To date, the only work which examines moving a task with fixed-function blocks to a new location where the blocks are not in the same position is Becker, et al. [17]. In this case, the blocks are not used, eliminating their need for migration. Unfortunately, given the diversity and abundance of fixedfunction blocks in contemporary FPGAs, simply ignoring and not using them is not a viable option for PRRs.

\section{Task Migration with Heterogeneous Blocks}

In this paper we describe FPGA routing architecture changes which allow tasks to be migrated so that fixed-function (hard) blocks are located in different locations within the target PRR. The approach does not require time-consuming placeand-route during the migration process. Since fixed-function blocks generally are aligned in vertical columns in contemporary FPGAs, we provide the ability for tasks to "float" horizontally within the logic array. The main contributions of this paper are:

- FPGA routing architecture enhancements which allow for routing connections to fixed-function blocks which are isolated from other routing in the FPGA routing fabric.

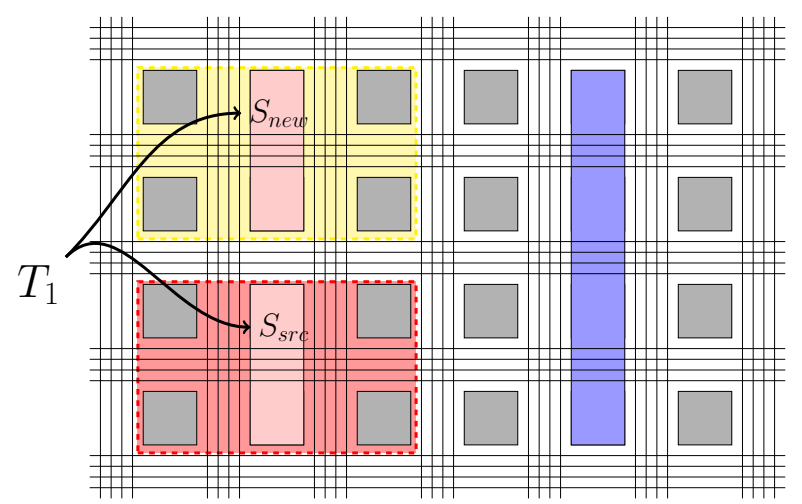

Fig. 1: Relocation problem in modern FPGAs: task $T_{1}$, placed on a set $S_{s r c}$ of resources, is only movable to another set $S_{\text {new }}$ with resource positions matching those in the original set

- An evaluation of the performance and area overheads incurred by this routing isolation for a collection of benchmark designs which include components mapped to both FPGA logic and fixed-function resources.

\section{A. Background}

To promote direct comparisons with contemporary FPGAs, we modify the well-known island-style FPGA model [18]. This architectural model (Figure 1) includes a two-dimensional array of logic blocks (LB) shown in grey squares that are interconnected via a programmable routing network. Our modeled LBs include a single four-input lookup table (LUT) and a flip flop (FF), although clusters of LUTs and FFs could also be used. The programmable routing consists of horizontal and vertical channels made of multiple wire segments. The channel segments can be connected to inputs and outputs of the LBs through connection boxes (CB). The channels are also connected to each other via switch boxes (SB). To optimize area and performance, modern FPGAs contain a spectrum of fixed-function blocks (shown as rectangles in Figure 1). These hardware resources reduce the demand for logic blocks for common functions, such as multiplication and bulk memory.

In an island-style FPGA, fixed-function blocks such as block RAMs and signal processing blocks (DSP blocks) are aligned in columns to simplify FPGA layout and the combining of multiple blocks into larger functional resources. Depending on block complexity, the height of a fixed-function block is often larger than a single grid location (e.g. the height of a logic block), as shown in Figure 1.

The allocation of fixed-function blocks across the logic fabric complicates task relocation for the shaded task which spans three columns in Figure 1. Given a hardware task placed at the position $\left(x_{\text {source }} ; y_{\text {source }}\right)$ in the array, it is limiting to find a position $\left(x_{n e w} ; y_{n e w}\right)$ in the logic fabric that contain a set of resources $S_{\text {new }}$ with all resources in exactly the same relative position as $S_{\text {source }}$. Overcoming the restrictions imposed by fixed-function resources requires making the routing connections to the fixed-function blocks more flexible. 


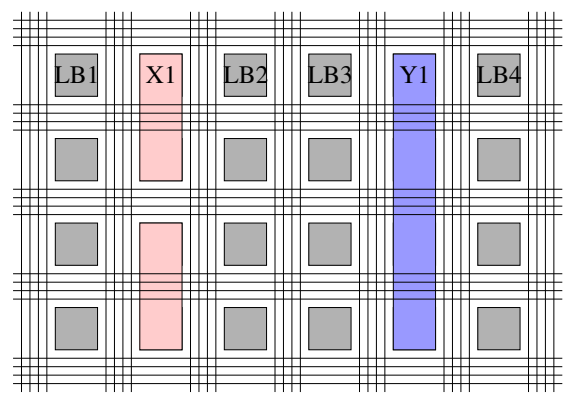

(a)

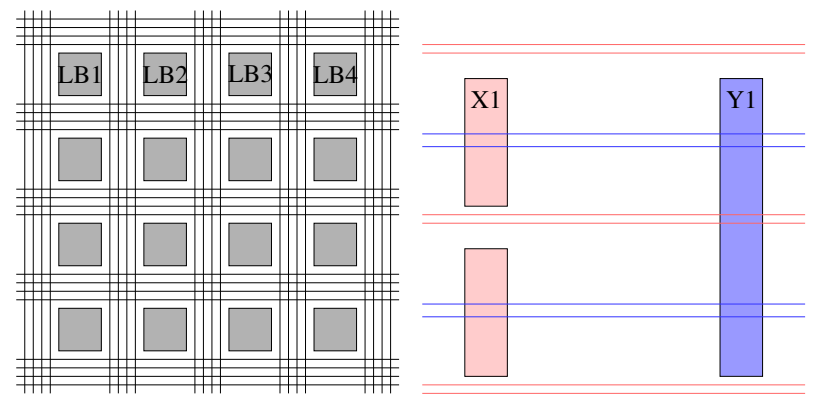

(b)

Fig. 2: Abstraction of heterogeneous resources: (a) Original logic array (b) Resulting set of logic-only and fixed-function only arrays

\section{B. Fixed-function blocks abstraction}

Increased routing flexibility for fixed-function blocks is introduced via an abstracted view of heterogeneous resources. Starting from the original architecture illustrated in Figure 2a, two separate logic resource layers are considered. The first layer, the logic array, includes the logic resources (LBs) of the architecture and its routing network, connection boxes and switch boxes. The second layer is made up of the fixedfunction blocks in the baseline logic array (e.g. memory blocks, DSP blocks, communication interfaces, etc.). The heterogeneous plane has its own routing network, composed of bidirectional routing long lines which span a defined region of the FPGA. Each of these lines can be connected to an input or output of the fixed function blocks. The fixed-function block input and output connections are restricted to their dedicated routing network. Links to the logic array are made via the heterogeneous-only long lines which connect to switch boxes of the logic array routing network. The extent of each of the long lines is optimized to provide good delay performance. The abstraction is presented in Figure $2 \mathrm{~b}$. It should be noted that this abstraction does lead to an increase in horizontal delay for routing in the logic-only (homogeneous) plane. Since the positioning on the die of fixed-function blocks is not known at place and route time, it must be assumed that such a block is physically located horizontally between each LB. The additional delay associated with the longer horizontal wires in the homogeneous plane must then be considered.

1) Routing network separation: The heterogeneous-only long lines only carry signals to or from fixed-function blocks. The remaining homogeneous routing network carries signals

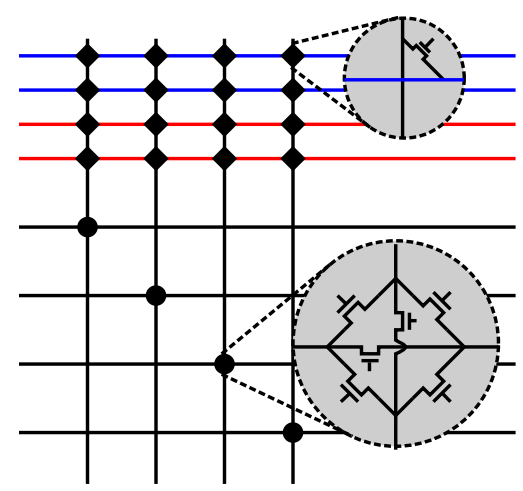

Fig. 3: Switch box enhanced to include connections between heterogeneous and homogeneous layers. Red and blue horizontal wires (the top four wires) represent heterogeneousonly long lines while the remaining black wires represent homogeneous routing

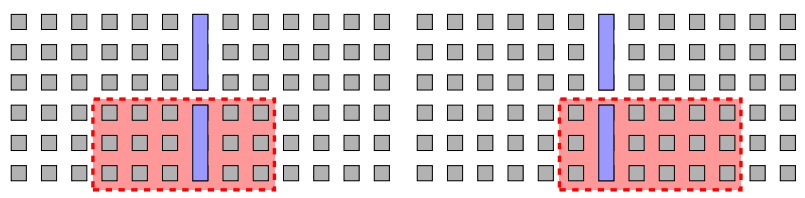

(a)

(b)

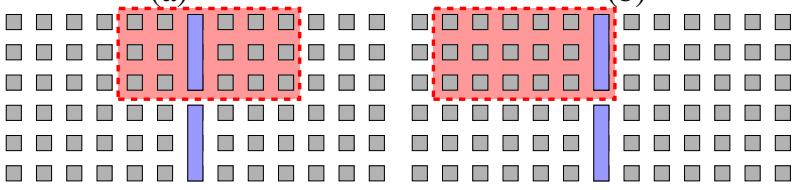

(c)

Fig. 4: Multiple relocation possibilities for the same bitstream of a task.

emanating from logic blocks. Heterogeneous-only long lines are connected to the homogeneous routing network through switch boxes at horizontal and vertical channel intersections, as shown in Figure 3. Routing segments spanning multiple LBs (e.g. 2, 4, 8, etc) are commonly found in island-style architectures. These segments do not make intermediate switch box connections to reduce wire delay. However, the heterogeneousonly long lines do connect to multiple adjacent switch boxes. Thus, it is possible to connect to these lines from multiple switch boxes. This additional level of routing allows for the ability to slide a given task horizontally along the horizontal heterogeneous-only long lines without affecting either layer of routing, as illustrated in Figure 4. Only the use of horizontal, heterogeneous-only long lines for fixed-function input and output signals was explored to avoid complex routing and excessive overhead for the overall circuit.

2) Partitioning: Since the size of fixed-function blocks can vary, the vertical and horizontal extent in LBs of a reconfigurable partition must be carefully selected. The width of the partition is bounded by the length of the heterogeneousonly long lines. The height of a partition is defined as the least common multiple of the height of the fixed-function blocks it contains. In this work, the FPGA partitions are identical in size and they are evenly distributed. The partitioning also improves 


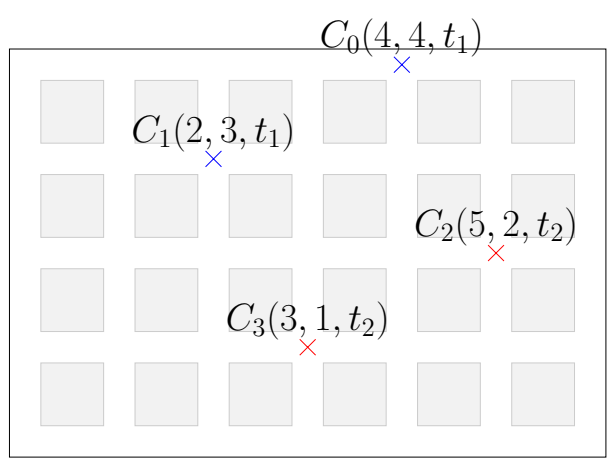

Fig. 5: Task model: each connection $C_{i}$ has to be linked to a fixed-function block via heterogeneous-only routing

the scalability of our approach: partitions can be duplicated to extend the size of the logic fabric.

3) Task model: In the context of this paper, we consider a hardware task to be a set of logic resources that are interconnected. A hardware task is described by a bitstream to be loaded into the configurable logic fabric. Unlike standard FPGA development where a hardware task contains an IP block that is loaded into the FPGA at a specific location, in this case a task is an aggregate of both interconnected homogeneous logic resources and a set of connections to heterogeneous-only long lines, as depicted by Figure 5. Each connection is characterized by its position $(x, y)$ within the task and its type $t$ is used to determine its resource connection. This set of connections is fixed, relative to the task itself, and the problem of relocation requires finding a corresponding match of fixed-function resources to this set. A task is defined as $T=\left\{C_{i}\left(x_{i}, y_{i}, t_{i}\right)\right\}$, where $x_{i}, y_{i}$ is the position of the connection and $t_{i}$ is its type.

\section{EXPERIMENTAL METHODOLOGY}

To experimentally evaluate the impact of our revised FPGA architecture on FPGA area and performance, the Versatile Place and Route (VPR) tool set (version 6.0) was modified. The following subsection details changes made to VPR for experimentation. In subsection IV-B, we describe the set of benchmark designs used for our experiments.

\section{A. Modeling in VPR}

The changes made to the VPR source code were made on the latest version ${ }^{1}$ available in the Verilog To Routing (VTR) [7] project repository.

1) Architecture: The VPR architecture description XML file that was used to model our circuit is based on k4_N10_memSize16384_memData8.xml, which is one of the default files shipped with the VTR tool flow. The file defines a simplified version of the Stratix IV architecture. The architecture was adapted to suit our needs and to provide a generic FPGA supporting multiple types of fixed-function blocks. The blocks include a $36 \times 36$ multiplier and a configurable dual-port RAM similar to a Stratix 144 Kbit embedded memory block. Figure 6 demonstrate two placements of the

\footnotetext{
${ }^{1}$ The checked out version was revision 3034 .
}
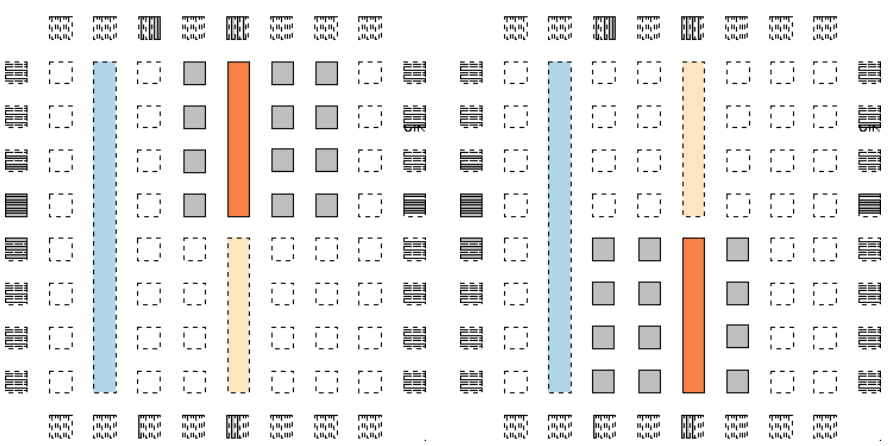

Fig. 6: Two placements of the same task on the enhanced architecture with VPR

same task with VPR and the enhanced architecture; routing was omitted for readability.

2) Routing abstraction: The key modifications to the VPR code base resulted in a dual-layer routing graph which allows inter-layer routing connections at switch boxes (Figure 3).

The segment types of the separated routing networks were modeled using two different segment type definitions in the architecture file. The first segment type, used for homogeneous routing, contains wires which span a single logic block. The second segment, used for heterogeneous-only routing, contains long lines spanning the whole chip. Since we restrict the routing to the fixed-function blocks to horizontal wires, the vertical routing adjacent to the fixed-function blocks is not used. In many VPR architecture definitions, a logic array block will be defined to have its inputs and outputs spread across its four sides. This behavior is undesirable in our case since inputs and outputs on left-hand and right-hand sides of the block will connect to vertical routing channels. As a result, we defined the location of input and output signals for fixed-function blocks as the top and bottom of the block.

Since VPR does not provide support for separate routing networks which are only interconnected in a few locations, modifications were made to the VPR routing graph generator. Source code was modified to enable the marking of segments of the architecture file segmentlist with an additional attribute, selective_routing. This attribute tags the segment as appropriate for either homogeneous signals (i.e. to or from a LB), heterogeneous-only signals (to or from a fixed-function block), or both types. Our model only uses heterogeneous-only long lines and segments which can carry both signal types. Additionally, the graph building procedures were adapted so that input and outputs of the complex blocks marked as heterogeneous are not routed on routing segments which are not tagged to route them. Similar marking was used for homogeneous routing segments.

In the original VPR release, long lines are balanced and their connections are rotated among the wires of the channels in which they reside. This behavior is desirable in an FPGA where one wants to leverage the full routability of the circuit. However, this rotation is not desirable in our model since connections to the heterogeneous-only long lines are more substantial. To circumvent this issue, we disabled the long line balance feature for segments marked as heterogeneous, 
TABLE I: VTR design flow benchmark suite

\begin{tabular}{lrrr}
\hline Benchmark & Memories & Multipliers & LBs \\
\hline LU8PEEng & 45 & 8 & 2,174 \\
bgm & 0 & 11 & 2,977 \\
boundtop & 1 & 0 & 272 \\
ch_intrinsics & 1 & 0 & 41 \\
diffeq1 & 0 & 5 & 43 \\
diffeq2 & 0 & 5 & 30 \\
mkDelayWorker32B & 41 & 0 & 497 \\
mkPktMerge & 15 & 0 & 17 \\
mkSMAdapter4B & 5 & 0 & 181 \\
or1200 & 2 & 1 & 273 \\
raygentop & 1 & 7 & 192 \\
stereovision1 & 0 & 38 & 990 \\
\hline
\end{tabular}

which allows the wires modeling the fixed-function routing to effectively span a fixed amount of the same columns. Currently, for a given defined segment, VPR will create both horizontal and vertical tracks in the routing graph. For our implementation, only horizontal long lines are used. Although vertical long lines for the heterogeneous-only routing layer were created, they were marked as unusable by the router and their area cost was ignored. All switch block and connection block connections to these wires were removed.

\section{B. Benchmarks}

Benchmarks with heterogeneous resources were used to evaluate our modified FPGA architecture. We choose to use the set of benchmarks included in the VTR design flow for experimentation. Among these benchmarks, 12 of them that use heterogeneous blocks (i.e. memories and multipliers) were selected to test our enhanced architecture. Table I sums up the benchmark details. The number of fixed-function blocks was calculated after the packing stage for our architecture. A total of 8 out of 12 designs make use of memories, and 7 out of 12 use multipliers. Of these designs, two of them have more than a thousand logic blocks.

\section{Results}

In this section we evaluate results obtained with the benchmarks described in Section IV-B using the enhanced architecture detailed in Section III. We use the modified version of VPR described in Section IV-A to perform timing-driven packing, placing and routing for the 12 designs. Comparative results are obtained with respect to a standard architecture which is similar to the enhanced one, with the exception of the new routing for the heterogeneous-only routing. Place and route data concerning the standard architecture are presented in Table II. The basic partition of both architectures is a $8 \times 8$ array of logic blocks with one column dedicated to a memory of 8 blocks height, and another column containing two multipliers of 4 blocks height. The results of the enhanced architecture were generated with multiple side-by-side horizontal partitions of size 8 since this is the smallest common denominator of the logic fabric. Each long line tied to a specific heterogeneous block spans a single partition.

\section{A. Logic array size}

As observed in Table III, our enhanced architecture has no influence on the minimal array size (in number of logic blocks) on which a task can be placed.
TABLE II: Place and route benchmark results on the standard architecture

\begin{tabular}{lrrr}
\hline Benchmark & Array size & Chan. width & Crit. delay (ns) \\
\hline LU8PEEng & 56 & 77 & 124.06 \\
bgm & 64 & 91 & 34.66 \\
boundtop & 20 & 36 & 7.17 \\
ch_intrinsics & 8 & 25 & 3.57 \\
diffeq1 & 12 & 32 & 16.17 \\
diffeq2 & 12 & 28 & 13.84 \\
mkDelayWorker32B & 50 & 65 & 11.25 \\
mkPktMerge & 32 & 24 & 5.60 \\
mkSMAdapter4B & 18 & 44 & 7.34 \\
or1200 & 25 & 55 & 13.36 \\
raygentop & 17 & 45 & 5.11 \\
stereovision1 & 37 & 76 & 6.24 \\
\hline
\end{tabular}

TABLE III: Place and route benchmark results on the enhanced architecture

\begin{tabular}{lrrrrrr}
\hline Name & Size & \multicolumn{2}{c}{ Chan. width } & H-wire & \multicolumn{2}{c}{ Crit. delay (ns) } \\
\hline LU8PEEng & 56 & 139 & $(1.81 \times)$ & 324 & 134.41 & $(1.08 \times)$ \\
bgm & 64 & 108 & $(1,19 \times)$ & 324 & 35.67 & $(1.03 \times)$ \\
boundtop & 20 & 56 & $(1.56 \times)$ & 324 & 7.51 & $(1.05 \times)$ \\
ch_intrinsics & 8 & 25 & $(1.00 \times)$ & 162 & 3.84 & $(1.08 \times)$ \\
diffeq1 & 12 & 96 & $(3.00 \times)$ & 234 & 15.96 & $(0.99 \times)$ \\
diffeq2 & 12 & 65 & $(2.32 \times)$ & 234 & 12.66 & $(0.92 \times)$ \\
mkDelayWorker32B & 50 & 117 & $(1.80 \times)$ & 324 & 11.46 & $(1.02 \times)$ \\
mkPktMerge & 32 & 92 & $(3.83 \times)$ & 324 & 4.84 & $(0.86 \times)$ \\
mkSMAdapter4B & 18 & 83 & $(1.89 \times)$ & 324 & 7.35 & $(1.00 \times)$ \\
or1200 & 25 & 77 & $(1.40 \times)$ & 324 & 13.62 & $(1.02 \times)$ \\
raygentop & 17 & 98 & $(2.18 \times)$ & 324 & 5.78 & $(1.13 \times)$ \\
stereovision1 & 37 & 129 & $(1.70 \times)$ & 324 & 6.97 & $(1.12 \times)$ \\
\hline
\end{tabular}

\section{B. Critical delay}

The critical delay of each circuit is expressed in nanoseconds in Tables II and III. To ensure an equal quality of results for both architectures, the same timing driven options were given to VPR, and the same routing seed was used in both cases. Most of the benchmarks sees a critical delay change by less than $10 \%$ between the standard architecture and the enhanced one.

The critical path delay increase in Table III is limited and results from routing congestion when vertical routing tracks in the homogeneous routing layer are used to reach the horizontal long lines and the increased capacitance of the long lines for short connections. Some of the benchmarks expose a decrease in critical path delay (in particular diffeq2, mkPktMerge). These improvements originate from the use of long lines to reach heterogeneous blocks, reducing the wire delay for signals in the critical path. The critical path timing of a task must consider relocation-related issues. Since the logic content of a relocatable task is placed relatively to the heterogeneous blocks, we do not know in advance where homogeneous signal will step over a fixed-function block. This issue is addressed at the place-and-route stage through the use of conservative timing.

In our case, the main area impact is a result of the distribution of long lines across the FPGA: a horizontal routing channel contains the minimum number of long lines required to route every I/O of a partition of the biggest channel (i.e. the amount of connections to the most connection-intensive fixedfunction block). Long lines which are not tied to a specific I/O in a horizontal channel can be used for other purposes, which 
can result in a reduced critical delay.

\section{Routing resources}

Our approach primarily focuses on routing network enhancements. The isolation of routing resources for logic-block only and fixed-function blocks has several implications. As described in Section III, each input and output of the fixedfunctions blocks is tied to a unique long line of a partition.

We observe an increase in the routing resources needed in the homogeneous routing network (the channel width in Table III), of $1.97 \times$ on average. Routing resources due to the routing heterogeneous-only long lines dedicated to fixedfunction blocks in the partition are shown in the $h$-wire column in Table III. It should be noted that the heterogeneous long lines ( $h$-wires) in the table for each benchmark are spread across an entire partition and not isolated in just one channel. As the number of $h$-wires increases, the number of logic-only homogeneous wires required to route signals up to the long lines increases to avoid network congestion.

Given these results, it is reasonable to define a nominal partition width of 8 columns, including heterogeneous blocks, for this specific architecture, as used in our experiments. This size allows for good performance in terms of flexibility, while still making a good trade-off in routing resources demand and occupied area. Our approach eliminates the need for routing when a task is moved. The only required change for the task bitstream is to shift the homogeneous-to-heterogeneous and heterogeneous-to-fixed-function block connections horizontally. The position of these connections in the bitstream can easily be offset by a fixed amount for the hundred or so connections for each fixed-function block as the task is loaded into the device.

\section{CONCLUSIONS AND FUTURE WORK}

This paper presents an enhanced FPGA architecture supporting the relocation of tasks and associated partial bitstreams even if the relative position of fixed-function blocks in the target partially-reconfigurable region is changed. A standard island-style FPGA architecture has been expanded to include a dedicated routing layer for fixed-function blocks. Long lines are used in this layer to allow blocks to "float" horizontally within a region, greatly expanding partially-reconfiguration region placement. The routing implications and costs associated with this freedom have been investigated for a variety of VTR benchmarks including fixed-function resources. This architecture is only the first step in the development of a dynamically reconfigurable FPGA for the FlexTiles project which allows for fast task migration and ease-of-use from a designer standpoint. The architecture treats I/Os in addition to multipliers and DSP blocks as fixed-function blocks, necessitating our new approach. Future implementations will allow for both horizontal and vertical connections in the heterogeneousonly routing layer. Additionally, research is needed to study the impact of various architectural parameters (e.g. task geometry, routing lines spreading) on detailed performance.

\section{ACKNOWLEDGMENT}

This research was sponsored by the European Commission under the 7th Framework program within the FlexTiles project
(FPT ICT-288248) and by the French Ministry of Research. R. Tessier was supported in part by National Science Foundation grant EECS-1201834.

\section{REFERENCES}

[1] L. Atieno, J. Allen, D. Goeckel, and R. Tessier, "An adaptive ReedSolomon errors-and-erasures decoder," in Proc. ACM/SIGDA International Symposium on Field Programmable Gate Arrays, 2006, pp. 150158.

[2] P. Sedcole, P. Y. K. Cheung, G. Constantinides, and W. Luk, "Runtime integration of reconfigurable video processing systems," IEEE Transactions on VLSI Systems, vol. 15, no. 9, pp. 1003-1016, 2002.

[3] D. Unnikrishnan, R. Vadlamani, Y. Liao, J. Crenne, L. Gao, and R. Tessier, "Reconfigurable data planes for scalable network virtualization," IEEE Transactions on Computers, vol. 62, no. 12, pp. 2476-2488, 2013.

[4] K. Compton, Z. Li, J. Cooley, S. Knol, and S. Hauck, "Configuration relocation and defragmentation for run-time reconfigurable computing," IEEE Transactions on VLSI Systems, vol. 10, no. 3, pp. 209 -220, 2002.

[5] J.-Y. Mignolet, V. Nollet, P. Coene, D. Verkest, S. Vernalde, and R. Lauwereins, "Infrastructure for design and management of relocatable tasks in a heterogeneous reconfigurable system-on-chip," in Proc. Design, Automation and Test in Europe Conference, 2003, pp. 986-991.

[6] F. Lemonnier, P. Millet, G. M. Almeida, M. Hubner, J. Becker, S. Pillement, O. Sentieys, M. Koedam, S. Sinha, and K. Goossens, "Towards future adaptive multiprocessor systems-on-chip: an innovative approach for flexible architectures," in Proc. International Conference on Embedded Computer Systems, 2012, pp. 228-235.

[7] J. Rose, J. Luu, C. W. Yu, O. Densmore, J. Goeders, A. Somerville, K. B. Kent, P. Jamieson, and J. Anderson, "The VTR project: architecture and CAD for FPGAs from Verilog to routing," in Proc. ACM/SIGDA International Symposium on Field Programmable Gate Arrays, 2012, pp. 77-86.

[8] G. Brebner, "A virtual hardware operating system for the Xilinx XC6200," in Field-Programmable Logic Smart Applications, New Paradigms and Compilers, 1996, pp. 327-336.

[9] Increasing Design Functionality with Partial and Dynamic Reconfiguration in 28-nm FPGAs, Altera Corporation, 2010.

[10] Partial Reconfiguration User Guide, UG702, Xilinx, Inc., 2013.

[11] A. Flynn, A. Gordon-Ross, and A. D. George, "Bitstream relocation with local clock domains for partially reconfigurable FPGAs," in Proc. Design, Automation and Test in Europe Conference, 2009, pp. 300-303.

[12] M. L. Silva and J. C. Ferreira, "Creation of partial FPGA configurations at run-time," in Proc. Euromicro Conference on Digital System Design: Architectures, Methods and Tools, 2010, pp. 80-87.

[13] J. Carver, N. Pittman, and A. Forin, "Relocation of FPGA partial configuration bit-streams for soft-core microprocessors," in Workshop on Soft Processor Systems, 2008.

[14] H. Tan and R. F. DeMara, "A multilayer framework supporting autonomous run-time partial reconfiguration," IEEE Transactions on VLSI Systems, vol. 16, no. 5, pp. 504-516, 2008.

[15] M. Touiza, G. Ochoa-Ruiz, E.-B. Bourennane, A. Guessoum, and K. Messaoudi, "A novel methodology for accelerating bitstream relocation in partially reconfigurable systems," Microprocessors and Microsystems, vol. 37, no. 3, pp. 358-372, 2012.

[16] Y. E. Krasteva, E. de la Torre, T. Riesgo, and D. Joly, "Virtex II FPGA bitstream manipulation: Application to reconfiguration control systems," in Proc. International Conference on Field Programmable Logic and Applications, 2006, pp. 1-4.

[17] T. Becker, W. Luk, and P. Y. Cheung, "Enhancing relocatability of partial bitstreams for run-time reconfiguration," in Proc. IEEE Symposium on Field-Programmable Custom Computing Machines, 2007, pp. 35-44.

[18] V. Betz, J. Rose, and A. Marquardt, Architecture and CAD for DeepSubmicron FPGAS. Boston, MA: Springer, 1999. 